\title{
Formation of Stability Lobe Diagram (SLD) for Chatter Free Milling on Aluminium Alloy
}

\author{
Gautam Kashyapi, Suhas Mohite ${ }^{*}$, Nachiket Belwalkar \\ Department of Mechanical Engineering, Government College of Engineering, Karad, India
}

Copyright $@ 2015$ Horizon Research Publishing All rights reserved.

\begin{abstract}
Chatter is a vibrational phenomenon which may cause damage of the tooling structure. The border between a stable (i.e., no chatter) and an unstable (i.e., with chatter) can be visualized in terms of the axial depth of cut as a function of spindle speed. The enveloping curve is a stability lobe diagram (SLD). Using SLD, it is possible to select cutting parameters to perform chatter-free cutting operations. In present study, an experimental methodology is followed based on tests in which a tapered workpiece allows gradual increase in axial depth of cut in feed direction, representing the $\mathrm{Y}$-coordinate of the SLD while speed of the spindle is increased for every pass representing $\mathrm{X}$-coordinate. Experiments are carried on Siemens 828D MTAB CompactMill machine. Acoustic sensor SKF CMAC 5084 is used to collect the milling sound. This work will allow operator to choose the combination of spindle speed and depth of cut for chatter free milling.
\end{abstract}

Keywords Stability Lobe Diagram, Aluminium Alloy

\section{Introduction}

The development of science, technology and technique has stimulated the expansion of manufacturing frontiers. Despite of all these advances, however, chatter vibration has been, for the last fifty years, a limitation to improving productivity and part quality in metal removal processes. Metal cutting processes can entail three different types of mechanical vibrations such as free vibrations, forced vibrations and self-excited vibrations. Free vibrations occur when the mechanical system is displaced from its equilibrium and is allowed to vibrate freely. Forced vibrations appear due to external harmonic excitations. Self-excited vibrations extract energy to start and grow from the interaction between the cutting tool and the workpiece during the machining process. This type of vibration brings the system to instability and is the most undesirable and the least controllable. Chatter is a self-excited vibration that can occur during machining operations and becomes a common limitation to productivity and part quality. Chatter is generally classified in two categories: primary and secondary. Primary chatter can be caused by the cutting process itself. Secondary chatter may be caused by the regeneration of waviness of the workpiece surface. Regenerative chatter is the most common form of self-excited vibration. It can occur often because most metal cutting operations involve overlapping cuts which can be a source of vibration amplification. Very early on, it was demonstrated that during a milling process chatter can arise at certain combinations of axial depth of cut and spindle speed (Figure 1). As a function of these two cutting parameters, the border between a stable cut (i.e. no chatter) and an unstable one (i.e. with chatter) can be visualized in a chart called SLD. The first approaches were presented by Tobias (1975) and Tlusty (1963). In some cases, the SLD of the system cutting tool, machine tool and workpiece is continuously changing and it is difficult to make predictions in advance and schedule the correct parameters to ensure stable operations. The methods based on chatter recognition do not need SLD identification. Quintana et al. (2008) developed a methodology in which a SLD shows the boundary between chatter-free machining operations and unstable processes, in terms of axial depth of cut as a function of spindle speed. These diagrams are used to select chatter-free combinations of machining parameters.. This paper deals with the phenomenon of chatter and its detection. In spite of the huge amount of investigation carried out regarding the chatter problem, small workshops operators, for several reasons, do not have access to apply the existent methodologies designed to reduce, suppress or avoid the chatter occurrence and its negative consequences. There is an important lack of knowledge, in workshops, regarding chatter troubles. Operators are not familiar with large analytical methods. These methodologies are usually too sophisticated and shop floor engineers and operators are not enough prepared to apply them. For these reasons, it seems interesting to develop simple experimental methodology to be easily applied in small and medium-sized workshops. Achieving the objectives will permit machine operators to improve the parameters selection and optimizing and increasing productivity while ensuring quality requirements. 


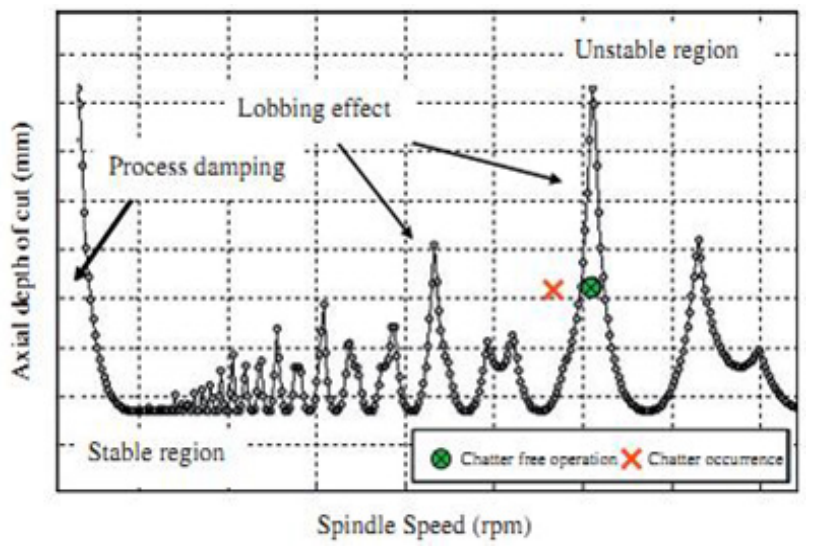

Figure 1. Stability lobe diagram courtesy Tobias and Fishwick

\section{Materials and Methods}

This dissertation deals with the phenomenon of chatter and its detection. In spite of the huge amount of investigation carried out regarding the chatter problem, small workshops operators, for several reasons, do not have access to apply the existent methodologies designed to reduce, suppress or avoid the chatter occurrence and its negative consequences. There is an important lack of knowledge, in workshops, regarding chatter troubles. Operators are not familiar with large analytical methods. These methodologies are usually too sophisticated and shop floor engineers and operators are not enough prepared to apply them. For these reasons, it seems interesting to develop simple experimental methodology to be easily applied in small and medium-sized workshops. Achieving the objectives will permit machine operators to improve the parameters selection and optimizing and increasing productivity while ensuring quality requirements.

The objectives of this dissertation work can be broadly stated as-

Gaining theoretical and hands-on knowledge about monitoring systems used on machines

Acquiring knowledge about sound pattern of milling operation

Performing experiments to obtain combination set of spindle speed and depth of cut for chatter free milling process

To establish SLD for chatter free milling process

Experiments are carried out on a Siemens 828D vertical milling machine (Figure 2) with spindle speed increasing from 200 to $2400 \mathrm{rpm}$ with an increment of $200 \mathrm{rpm}$ per pass. The operation consisted of slots along $\mathrm{X}$ axis of the machine tool while increasing the axial depth of cut in $\mathrm{Y}$ direction. The feed rate is maintained constant with $0.1 \mathrm{~mm} / \mathrm{rev}$. No coolant was used. The material used was aluminum alloy 6082 .

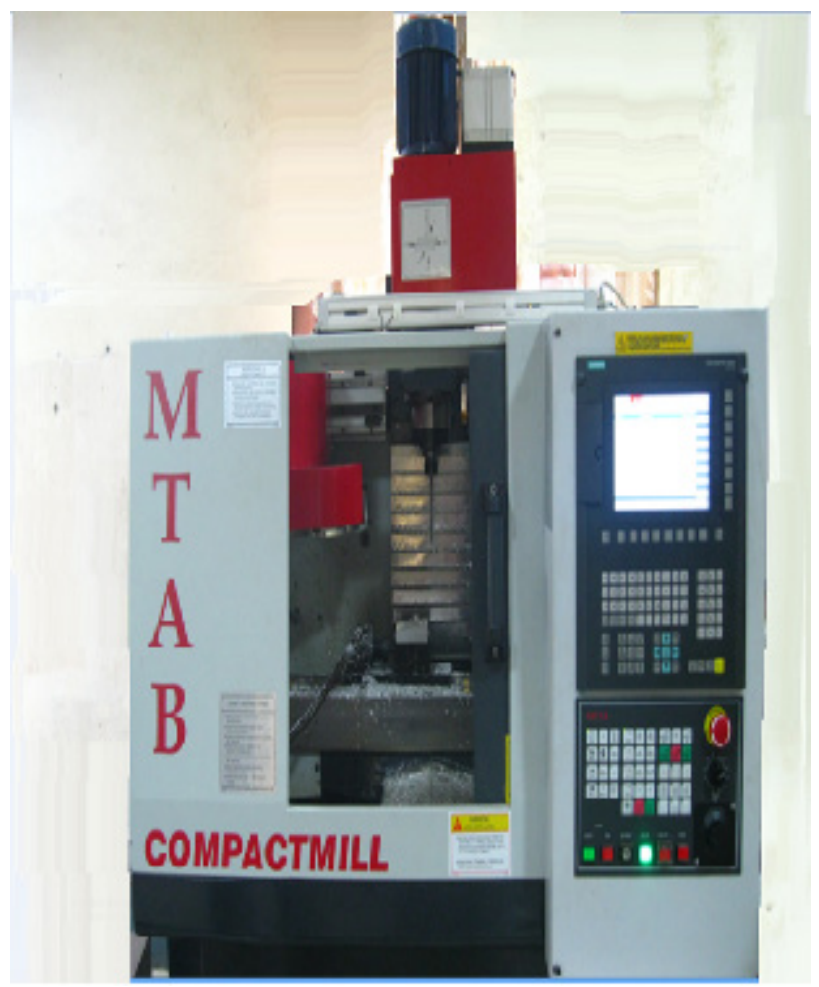

Figure 2. Siemens 828D milling machine

The methodology for conducting experiments and analyzing results is outlined below.

Collection of acoustic emission signals from the milling process using AE sensor and/or microphone for combination of spindle speed and depth of cut on work piece.

FFT analysis of signal generated during milling process for determination of SLD and chatter.

Collecting different parameters like sound amplitude, sudden change in peaks during successive machining for the given combination of spindle speed and depth of cut of work piece.

Result will reveal the suitability of selected combination of spindle speed and depth of cut for chatter free milling operation.

The sensors used for the experimentation are mounted as shown in Figure 3. The acoustic sensor is held as near as possible to the machining process using dial indicator stand used for the experiment. The cutting tool used for experiment is flat end mill, $8 \mathrm{~mm}$ diameter and $109 \mathrm{~mm}$ length with two cutting edges. The instrument used for acquiring the signals generated during the operation is shown in Figure 5. The SKF Microlog Analyzer Pro ${ }^{\circledR}$ (see Figure 4(a)), is used to generate the FFT of the acoustic signals collected using acoustic sensor SKF CMAC 5084 (see Figure 4(b)). 


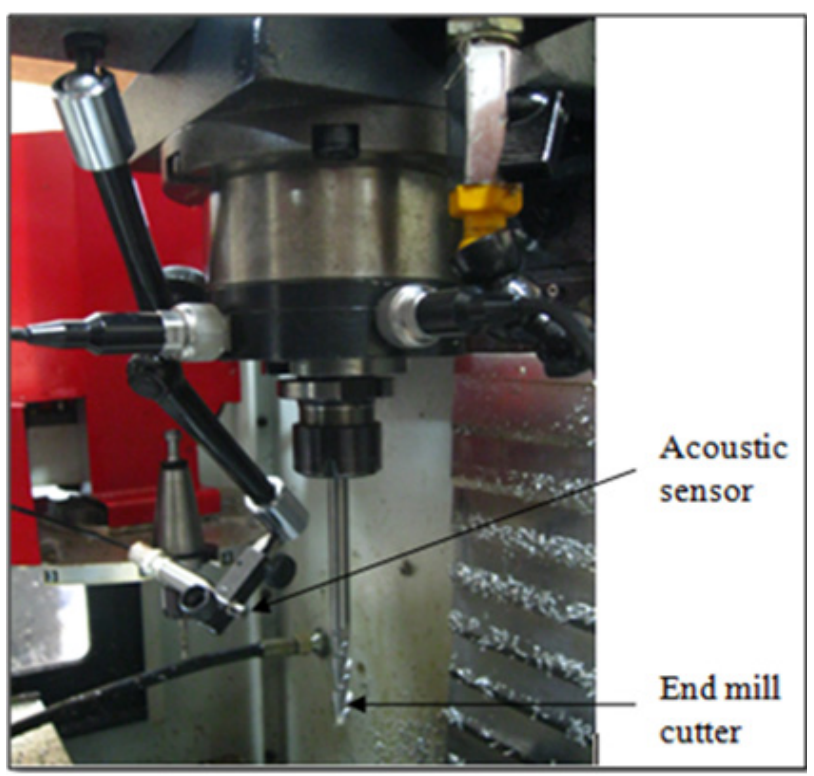

Figure 3. Mounting of acoustic sensor

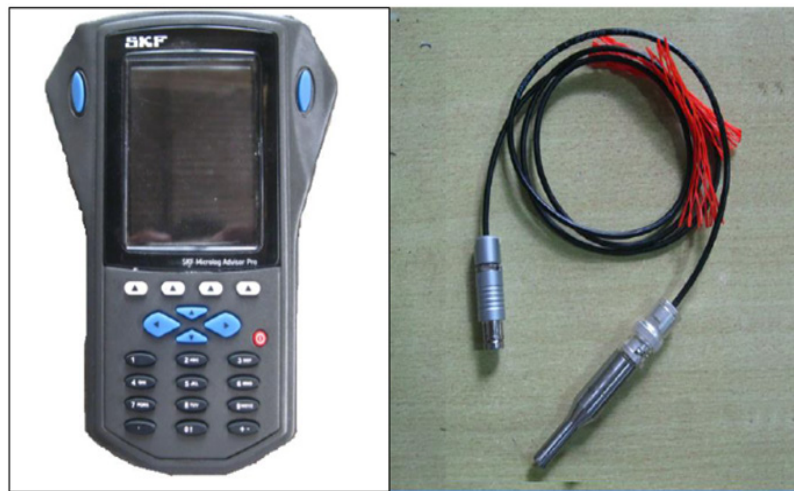

(a)

(b)

Figure 4. (a)SKF Microlog Analyzer Pro® (b) Acoustic sensor SKF CMAC 5084

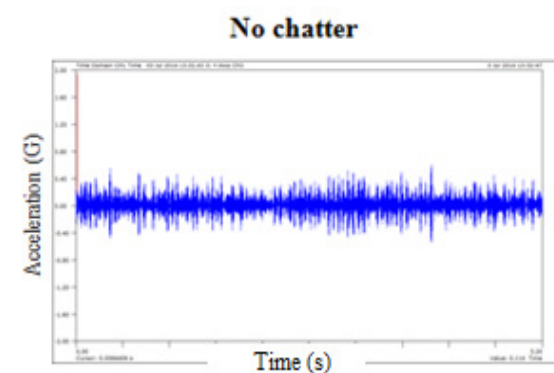

(a)

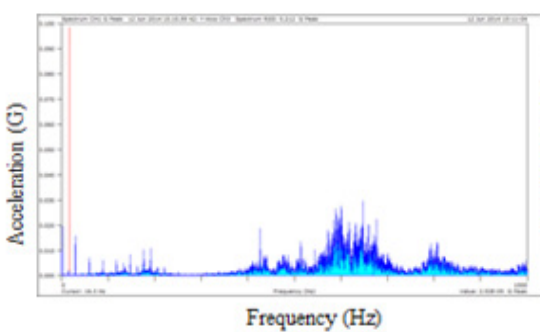

(c)

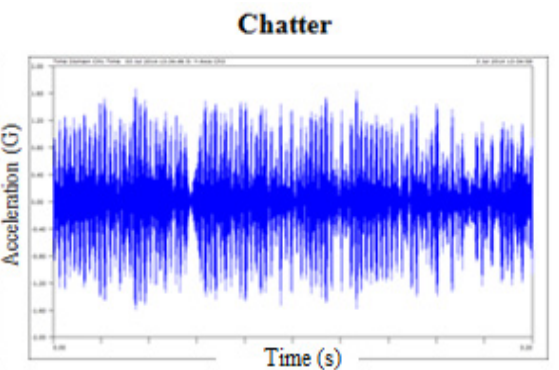

(b)

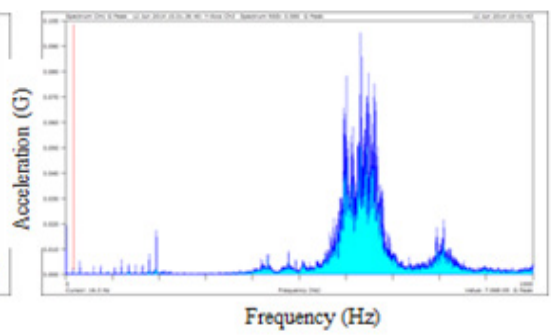

(d)

Figure 5. (a).Time domain of acoustic signature for $1000 \mathrm{rpm}$ spindle speed and $0.6 \mathrm{~mm}$ depth of cut, (b) Time domain spectrum for $1000 \mathrm{rpm}$ spindle speed and $0.8 \mathrm{~mm}$ depth of cut, (c) and (d) Frequency spectrum of Figure 4(a) and (b), respectively 


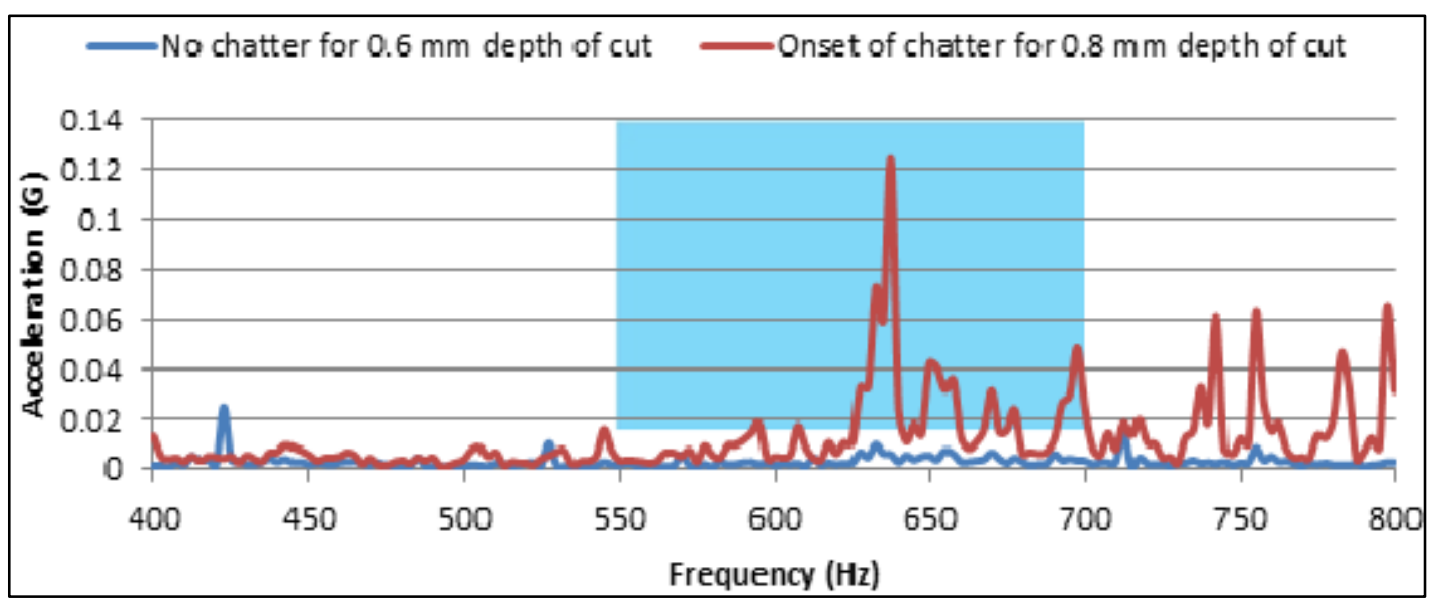

Figure 4. Comparison of frequency spectrums for with and without chatter at $1400 \mathrm{rpm}$ spindle speed

Table 1. Combination of spindle speed and maximum depth of cut for aluminum alloy 6082

\begin{tabular}{|c|c|c|c|c|c|c|c|c|c|c|c|c|}
\hline $\begin{array}{c}\text { Spindle speed } \\
\text { (rpm) }\end{array}$ & 200 & 400 & 600 & 800 & 1000 & 1200 & 1400 & 1600 & 1800 & 2000 & 2200 & 2400 \\
\hline Depth of cut (mm) & 0.4 & 0.6 & 0.6 & 0.6 & 0.8 & 1.0 & 0.8 & 0.4 & 1.0 & 1.4 & 2.6 & 0.8 \\
\hline
\end{tabular}

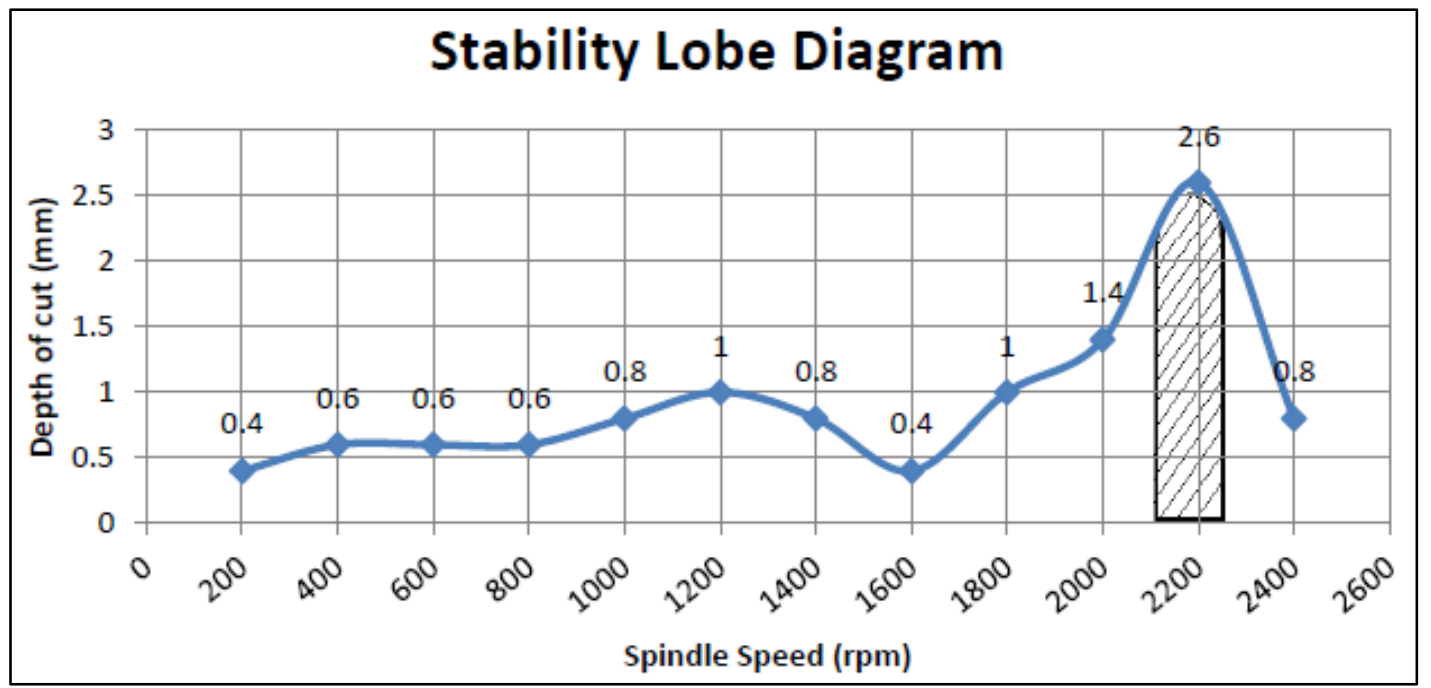

Figure 5. SLD for aluminum alloy 6082 , region below the graph indicates chatter free operation and the hatched area correspond to maximum MRR

\section{Results}

Chatters can be identified by viewing FFT spectrum around the natural frequency of the end mill cutting tool used for the operation. The natural frequency of the tool is $560 \mathrm{~Hz}$. Figure 5 shows time domain and FFT domain spectrum for aluminum alloy 6082 for $1000 \mathrm{rpm}$ spindle speed.

Figure 4(a) shows the stable condition for $0.6 \mathrm{~mm}$ depth of cut and figure 4(b) shows unstable cutting conditions for $0.8 \mathrm{~mm}$ depth of cut. It is seen that, amplitude for unstable cutting condition has increased compared to stable condition but, it is difficult to identify whether the rise in amplitude for unstable condition is due to chatter. Now, this time domain signature is converted to frequency domain. Figure 5(c) and (d) shows FFT of figure 5(a) and figure 5(b), respectively. Here, we can see rise in amplitude for unstable condition within the range of $550 \mathrm{~Hz}$ to $700 \mathrm{~Hz}$ in which natural frequency of the tool lies.

Figure 6 shows the FFT obtained for spindle speed of $1400 \mathrm{rpm}$. The depth of cut increases gradually in feed direction. Blue line shows the spectrum for $0.6 \mathrm{~mm}$ depth of cut while red line shows the spectrum for $0.8 \mathrm{~mm}$ depth of cut where the onset of chatter is observed. As it is clearly seen that, there is sudden rise in peaks for red spectrum between $550 \mathrm{~Hz}$ to $700 \mathrm{~Hz}$ as compared to blue spectrum thus, it indicates chatter. The highlighted region in graph helps in identifying chatter. Machining is stopped immediately as spectrum passes through unsafe region. Table 1 shows maximum depth of cut for corresponding spindle speed. 


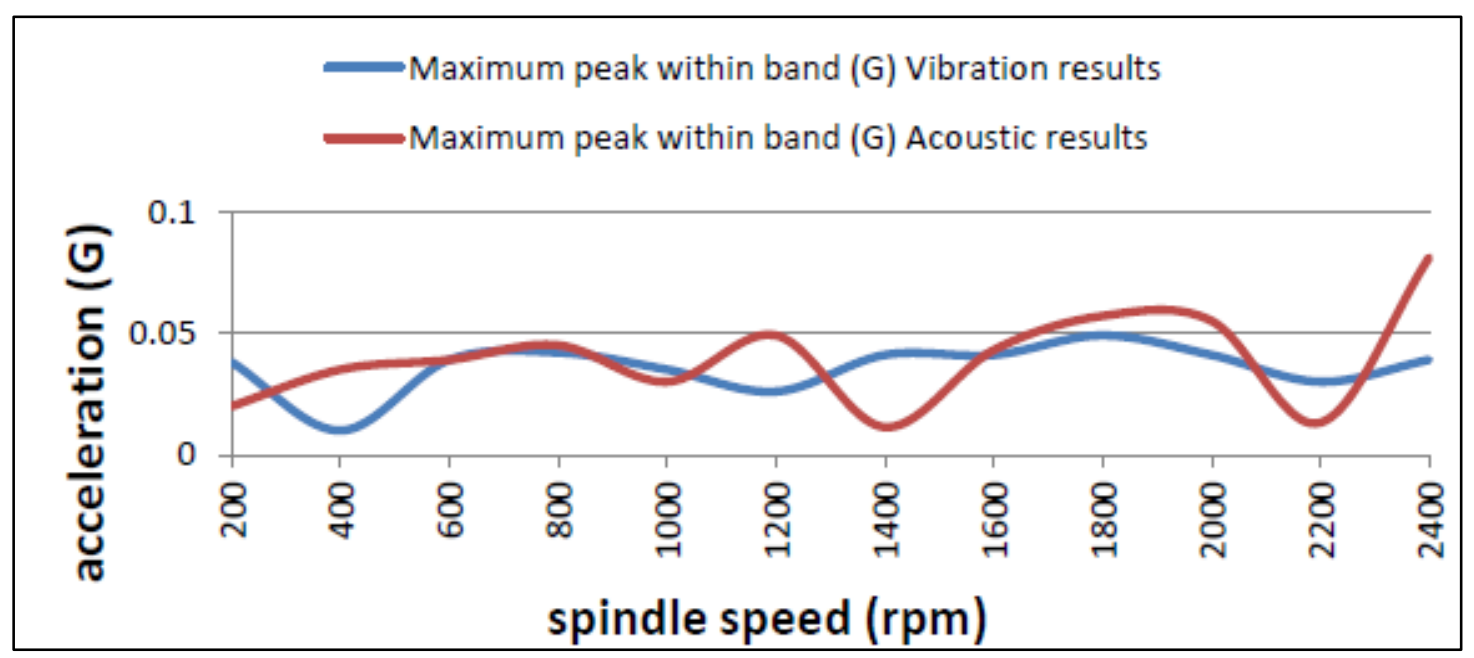

Figure 6. Comparison of peaks obtained using vibration and acoustic analysis for AA-6082

Figure 7 shows that the SLD obtained for the AA-6082 with the help of acoustic sensor. In the rpm range of 2100 to $2300 \mathrm{rpm}$ maximum MRR $(14 \mathrm{cc} / \mathrm{min})$ is possible with a depth of cut of about $2.5 \mathrm{~mm}$.

\section{Discussion}

Figure 8 shows the comparison of maximum peaks obtained using acoustic sensor and accelerometer in the band of $550 \mathrm{~Hz} 700 \mathrm{~Hz}$. Figure shows that, there is not much difference.

\section{Conclusions}

From all these experimental observations and subsequent analysis of results, following conclusions are drawn.

- The SLD is obtained for AA-6082 using acoustic sensor technique. It is seen that maximum MRR is obtained at a depth of cut $2.6 \mathrm{~mm}$ for spindle speed of $2200 \mathrm{rpm}$. For chatter free operation, MRR comes to be $14 \mathrm{~cm}^{3} / \mathrm{min}$.

- Concerning chatter phenomenon there is no linear relation between the spindle speed and depth of cut.

- The region below graph is chatter free. Use of spindle speed and corresponding depth of cut ensures chatter free operation leading to acceptable surface finish.

This work will allow operator to choose, or limit the combination of spindle speed and depth of cut for chatter free milling process.

\section{Acknowledgements}

This work is carried out in the Computer Integrated Manufacturing Laboratory, Department of Mechanical Engineering, Government College of Engineering, Karad. The Lab. is set up under Technical Education Quality
Improvement Programme (TEQIP-II).

\section{Appendix}

Tool specifications

\begin{tabular}{|c|c|c|}
\hline Sr. No. & Description & Specification \\
\hline 01 & End mill tool material & Tungsten carbide \\
\hline 02 & No. of flutes & 02 \\
\hline 03 & Diameter of tool (d) & $8 \mathrm{~mm}$ \\
\hline 04 & Helix angle & $45^{\circ}$ \\
\hline
\end{tabular}

In this experiment the material of the tool is tungsten carbide. The natural frequency of tool is calculated as follows,

$$
f_{n}=\frac{1}{2 \pi} \alpha_{n}^{2} \sqrt{\frac{E I}{m L^{3}}} .
$$

For, $\mathrm{L}=0.109 \mathrm{~m}, \mathrm{~d}=0.008 \mathrm{~m}, \rho=15630 \mathrm{~kg} / \mathrm{m}^{3}$

$$
\begin{gathered}
I_{z z}=\frac{\pi}{64} d^{4} \\
I_{z z}=\frac{\pi}{64} 0.008^{4} \\
I_{z z}=2.01 \times 10^{-10} \mathrm{~m}^{4} \\
V=\frac{\pi}{4} d^{2} L \\
V=\frac{\pi}{4} \times 0.008^{4} \times 0.109=5.47 \times 10^{-6} \mathrm{~m}^{3} \\
m=\rho V
\end{gathered}
$$

Substituting in Eq. (1), we get 


$$
\begin{gathered}
\therefore f_{1}=\frac{1}{2 \pi}(1.875)^{2} \sqrt{\frac{550 \times 10^{9} \times 2.01 \times 10^{-10}}{0.0856 \times 0.109^{3}}} \\
\therefore f_{1}=558.76 H z
\end{gathered}
$$

\section{REFERENCES}

[1] Tobias S., Fishwick, Theory of regenerative machine tool chatter, The engineer 1958258, 1975.

[2] Tlusty J. ,The stability of machine tools against self-excited vibrations in machining. International Research in Production Engineering , 465-474, 1963.

[3] Quintana G.,Teixidor D., A new experimental methodology for identification of stability lobes diagram in milling operations. International Journal of Machine Tools \& Manufacture, 48,1637-1645, 2008 